\title{
Effective Visible Light Communication System for Underground Mining Industry
}

\author{
Ibrahim Mansour \\ Al-Ahliyya Amman University
}

\begin{tabular}{l} 
Article Info \\
\hline Article history: \\
Received Dec 9, 2019 \\
Revised Jun 18, 2020 \\
Accepted Jun 27, 2020 \\
\hline
\end{tabular}

Keyword:

Light Fidelity (Li-Fi)

Visible Light Communication

(VLC)

Underground Mines

Optical communication systems

\begin{abstract}
Adequate lightening and efficient communication technology have prime importance for safe underground mining communication system operations. Existing conventional light and communication systems used in underground mines are not very efficient solutions due to heavy power and maintenance requirements. Also, efficient communication technology is required for instantaneous reporting of any potential disaster event under hazardous underground environment. In this paper, we propose light fidelity ( $\mathrm{Li}-\mathrm{Fi})$ as an efficient way of incident reporting as well as source of illumination for mines. Visible light communication (VLC) system is being used in mines operations, to support communication-blind areas. It exhibits superior performance over traditional radio frequency (RF) communication systems, in terms of low energy consumption, higher data rates achieved, and wide frequency band (430-790) THz. In this work, we present VLC system for safe and reliable mining operations and analyze and discuss corresponding channel impulse response (CIR). We consider effect of shadowing and dust on our optical channel model. We compare the performance of our system with available methods in terms of bit error rate (BER) and CIR and prove the superiority of our proposed system.
\end{abstract}

Copyright (c) 2018 Institute of Advanced Engineering and Science. All rights reserved.

\section{Corresponding Author:}

Ibrahim Mansour

Al-Ahliyya Amman University,

Amman-Jordan 19328.

Email: imansour@ammanu.edu.jo

\section{INTRODUCTION}

To improve health and safety at work and to reduce the rate of accidents, modern technologies have been widely adopted and used. Underground mining is one of the most important and useful systems for exploration under earth's assets and miners work under dangerous and hazardous environments. Many accidents are possible such as cave ins, gas explosions, chemical leakages, electrocution, and fires. Miners can get lost in mines under disaster situations, and should be warned, located and rescued as quickly as possible. The most important issue in underground mining industry is to keep information flow maintained in both directions during mining operations. Information such as measuring the atmosphere of the mine, detecting hazardous gases and smoke, monitoring mining machinery, and, most important of all is monitoring and locating of miners in case of any accident. Various wireless technologies have been recommended for this purpose, but all comes with some specific limitations. For instance, higher costs and storage requirements for Bluetooth, electromagnetism and requirement of higher electronic energy for WLAN, and harm caused to miner's eyes in case of infrared systems are few to mention.

Visible light communication (VLC) and its Application in Underground Mining: VLC has gained lots of attention for providing reliable communication facilities in indoor and outdoor environments. VLC can be used as a new potential to improve the underground mining wireless communication systems. It is comprised of LED based system, therefore can provide higher data rates over short distance, compared to RF technologies suffering from electromagnetic interferences. VLC technology does not only improve response time (use of on-off keying (OOK) modulation technique for underground operations) but also provide guaranteed communication availability [1]. It is a strong candidate for replacement of conventional systems 
for being cost effective, license free and usage of inexpensive LEDs. In contrast to incandescent and fluorescent lamps, LED lights have longer lifetime and higher luminance efficiency with proved lower operating voltage and power consumption.

Physical layer of VLC is well investigated in terms of channel modeling and adaptive filtering, which are the foundations for the reliable and robust VLC system design. There has not been much work done in channel modeling for underground VLC link, therefore intensive research and investigation is required for practical adaptation in real life applications.

Our proposed system presents an efficient VLC enabled solution for rescuing workers and equipment inside mines. We calculate total received power and evaluate the bit error rate (BER) of our system. This paper is organized as follows: In section 2, we discuss application of VLC in underground mines followed by associated challenges in section 3. Section 4 discusses the proposed system and channel model for underground mines. Simulation results and corresponding analysis is provided in section 5, followed by conclusions in section 6 .

\section{VISIBLE LIGHT AND MINING INDUSTRY}

Due to their low energy consumption and small size, VLC systems have been widely used in underground mining industry. In addition of using VLC in underground mines wireless communication, visible light-based positioning systems are also widely used. Once LED lighting system is installed, VLC based positioning can provide a seamless positioning service within much larger coverage area compared to current techniques.

A. $\mathrm{Li}-\mathrm{Fi}$ versus $\mathrm{Wi}-\mathrm{Fi}$

Both Wi-Fi and Li-Fi can transmit data over electromagnetic spectrum; Wi-Fi utilizes radio waves while Li-Fi utilizes visible light. High capacity Li-Fi spectrum is an excellent substitute to capacity bottleneck Wi-Fi. As visible light spectrum is 10,000 times larger than the entire radio frequency spectrum. $\mathrm{Li}-\mathrm{Fi}$ is a high intensity light source providing clean lighting solutions for general and special lighting. Li-Fi applications exhibit energy efficient long lifetime, and both full spectrum and dimming characteristics. Also, $\mathrm{Li}-\mathrm{Fi}$ achieves data rates up to $10 \mathrm{Gbps}$, that is 250 times more than fastest $\mathrm{Wi}-\mathrm{Fi}$.

In addition to above, Li-Fi can be used in electromagnetic sensitive areas such as aircraft cabins, hospitals and nuclear power plants without causing electromagnetic interference. Last but not the least, Li-Fi is a cheap and environment friendly solution compared to Wi-Fi.

B. Traditional Communication Systems

Conventionally, different systems have been used for communication for underground mining systems. There are many wireless systems such as Wi-Fi (IEEE 802.11), Bluetooth (IEEE 802.15) and WiMax being used in underground mines application. Ultra-Wide Band system is another radio system for short range communication with very low power and very high data rates [2]. Magneto phones, paging phones and trolley carrier phones [3,4] are few other options to mention here. However, Magento phones has limitations of being operated with DC batteries and AC signals. While Paging phones are partially wired system with no tracking capability.

One of the most well-known communication systems for underground mines is "Through the Earth (TTE)" system. This system provides alarming, tracking and messaging services by transmitting low frequency signals to cap lamps by using loop antennas [5]. The biggest drawback for TTE system is the radio wave attenuation, which depends on the frequency of radio wave, earth conductivity, transmitter power, antenna type and noise over the surface [6].

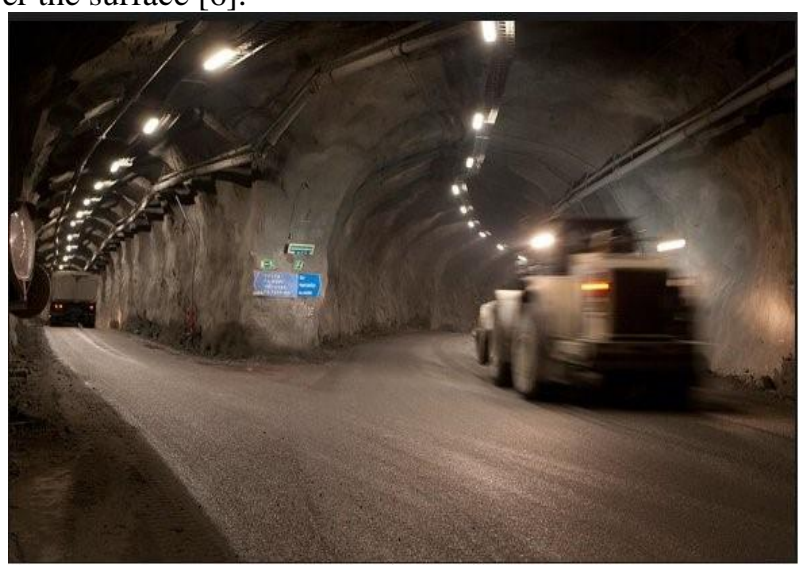

Figure 1. LEDs arrays in underground mine 
Radio Frequency Identification Device (RFID) system is also recommended for mining operations. These are comprised of small chips with RF identifier tags, readers, and host station. RFID tags are of two types, active and passive. Signal range of active tags is 100 meter and that of passive is 6-8 meters. Active tags are being used in underground mining applications due to extended allowed communication range [7].

\section{VLC for Positioning System}

VLC based positioning is a technique that uses visible light signals for determining the location of mobile devices and it has many advantages over RF based positioning. VLC based positioning systems can be installed inexpensively since it uses existing lighting systems with some modifications. Visible light positioning can be safely used in many places where RF cannot be used, such as underground mines. Another major advantage of VLC based positioning is visible light is less prone to multipath fading effects thus making optical signal more predictable [8].

\section{VLC for Wireless Communication in Underground Mines}

In general, underground mines consist of mining roadway and mine working face. Most of the previous works considered the horseshoe shape for mining roadway and a rectangle chamber for working face [9].

- Mining roadway: Mining roadway is a universal term used for shafts, tunnels, and chambers. It is usually used to transport the product (coal, gold, ... etc.) by miners and machinery. In addition, its shape can be divided into square, horseshoe-shaped, rectangular and trapezoid. The roadway is narrow with short-distance visibility. Moreover, this type is not regular due to irregularities in the shapes of walls and ceiling.

- Mine working face: Mine working face is rectangular shaped large sized mechanized equipment and used by miners to get the product from beneath the ground. During the extraction process, the electromagnetic radiation is generated, and some radio waves are absorbed by water vapor and flammable gases in working face. Therefore, RF wireless communication is seriously interfered by electromagnetic waves in underground mining.

(1) Communication Scenarios: Usually, communication system in underground mines consists of wireless communication technology that utilizes ultra-low frequency band (300-3000) $\mathrm{Hz}$ and power line carrier (PLC) communication technology. Therefore, VLC can utilize the integrated PLC equipment to maintain communication between ground and the underground mining. Hence, a VLC link can be built between a light base station and a miner. The LED lamp can be fixed on the top of the horseshoe mining roadway, mine working face and the helmets of miners, which are considered as the base stations to transmit information in light form. The optical receiver $(\mathrm{Rx})$ can be fixed on the miners' helmets to receive information. The two main communication scenarios that are applicable in underground mines communications systems (UMCs) are: Miner-to-miner (M2M) communication and Infrastructure-to-miner (I2M) communication.

- Miner-to-miner (M2M) communication: The miner can wear a LED lamp as a mobile end user in the mine. The human eye perceives only the average intensity when light changes fast enough. Therefore, LEDs can transmit data without a noticeable effect on lighting output or human eyes. Miners can communicate with each other by LED lamp that is fixed on their mine helmets. The miners use the light to transmit information about the mine environment and their location to other miners. Therefore, VLC channel modeling for the M2M scenario can be characterized by considering only the Line-of Sight $(\mathrm{LoS})$ propagation within the underground mining environment [9].

- Infrastructure-to-Miner (I2M) communication: In this communication scenario, the LED lamp that is fixed on mine wall is regarded as light source and information source to provide both illumination and communication. It acts as an optical wireless base station. LEDs can transmit data by changing switches status fast enough. So, human eyes cannot notice it. The miners can receive information by the mine helmets. Unlike M2M, the channel model of I2M can be characterized by considering both LoS and Non-line-of-sight (NLoS) propagation within the mine environment [9].

E. Lighting Requirements in Underground Mines

Lightening in underground mine is very important for illumination purposes, and the distribution of LEDs is also important for uniform lightning. According to [10] underground mining lighting standards, minimum requirement for lighting power density is $0.158(\mathrm{~W} / \mathrm{m} 2)$ and minimum illuminance is 107.65. These standards are considered minimum requirements for both lighting and signal communications. To avoid blind spots and make uniform light distribution of the receiving plane, it is important to design the LEDs lightening system optimally. 


\section{UNDERGROUND VLC CHALLENGES}

Various challenges are associated with optical signal communication for underground mining operations. Multipath fading, optical signal dispersion and interference due to dust particles play vital role in signal degradation. In the following paragraphs, we discuss most prominent factors affecting reliable optical communication.

A. Shadowing

Since the underground mines usually have large numbers of equipment and workers, increase in probability of shadowing is noticed. Effect of shadowing on VLC system is severe due to its line of sight propagation nature and hence, shadowing can seriously impair the system performance [11].

In general, there are three types of shadowing, mild, moderate and severe. Shadowing effect for visible light can be modelled by a Bimodal Gaussian distribution [12].

The Bimodal Gaussian distribution effectively represents separate propagation modes caused by different polarizations.

The Bimodal distribution has the following probability density function (PDF) [13]

$$
f\left(z, \mu_{0}, \sigma_{0}, \mu_{1}, \sigma_{1}\right)=\frac{1}{2 \sqrt{2 \pi\left(\sigma_{0}\right)^{2}}} \exp \left(\frac{-\left(z-\mu_{0}\right)^{2}}{2\left(\sigma_{0}\right)^{2}}\right)+\frac{1}{2 \sqrt{2 \pi\left(\sigma_{1}\right)^{2}}} \exp \left(\frac{-\left(z-\mu_{1}\right)^{2}}{2\left(\sigma_{1}\right)^{2}}\right)
$$

where $\mu_{0}, \sigma_{0}, \mu_{1}, \sigma_{1}$ are the means and the standard deviations of the first and the second Gaussian distributions, respectively.

Optical diffraction phenomenon is used to overcome the effect of shadowing, by employing a receiver with a wide field of view (FOV). In this work, we simulate the probability of error for low, moderate and severe shadowing conditions.

\section{B. The Effects of Mine Dust Particles on Optical Signal}

Dust suspended in air is generated during mining operations of crushing, tunneling, extraction, and transportation. The generated dust particles diffuse gradually with the mine ventilation system, polluting the entire mine atmospheric environment. This dust is considered a major security disaster during mine production because of the explosion hazard when the dust concentration reaches a specific value. So, it is critical to monitor and obtain information about dust concentration variations [14].

These dust particles have tendency to absorb electromagnetic and light waves, hence resulting into optical signal attenuation due to scattering and absorption. The relation between incident light intensity and transmission light intensity can be expressed as follows [15]:

$$
I=I\left(\alpha_{T}, \beta_{T}\right) e^{\frac{-1.5 C L K_{e}}{d}}
$$

where $I\left(\alpha_{T}, \beta_{T}\right)$ is the initial light intensity, $C$ is the number of suspended particles in unit volume $\left(\mathrm{cm}^{-3}\right), L$ is light path $(\mathrm{cm})$, and $K_{e}$ is light attenuation coefficient: $K_{e}=Q_{s c a}+Q_{a b s}$, and $Q_{s c a}, Q_{a b s}$ are scattering coefficient and absorption coefficient, respectively. The light attenuation coefficient: $K_{e}$ is given by [16]

$$
K_{e}=0.993 e^{-2.01 C}
$$

\section{Noise}

Noise generated due to mining equipment inside the underground mine degrades the signal quality. External or internal noise reduces the coverage range of the communication system. Electric motors, power lines and lighting systems are some major sources of noise in a mine tunnel. The performance of the communication system is highly affected by noise level. Noise caused by different appliances, cable lines, electric motors and mining equipment are in the frequency bands in which underground communication devices operate [17].

D. Multi-path Fading

Due to the presence of various underground mining equipment, and other reflectors multipath propagation comes into the picture. This causes fluctuations in signal power and fading in signal strength [18].

\section{E. Propagation Velocity}

A wave propagating in clean air has a stable propagation velocity compared to waves propagating in hinder environments, as it adds the dielectric effects. Dielectric property of the medium changes with change 
in temperature in underground mine. Due to this, a signal propagating inside the mine is distorted and this leads to a degradation in system performance [18].

\section{SYSTEM MODEL}

In this section, the proposed model for VLC enabled underground communication system is discussed. The proposed system is based on VLC and intensity modulation and direct detection (IM/DD) technique.

1) System and Operational Framework: It consists of ground surface part and underground part for underground environment, as shown in Fig. 2. Furthermore, the underground component includes arrays of LEDs used for lighting the underground mine, and for transmitting data coming from workstation to the miners using miner's lamp. Moreover, arrays of photo diodes (PDs) are used as receivers, when the miners send their signals to the workstation. Another important part of the underground components is the miner's lamp, used as an as optical data transceiver. When workstation wants to send some warning signals to miners, firstly the signal is transmitted to LED through PLC, then it is sent from VLC transmitter (LEDs) to VLC receiver (PDs) at the miners' lamps through optical signal.

In the uplink path, when the miners face any hazardous conditions, the transmitter on the miner lamp sends a signal to the VLC receiver as shown in Fig. 2.

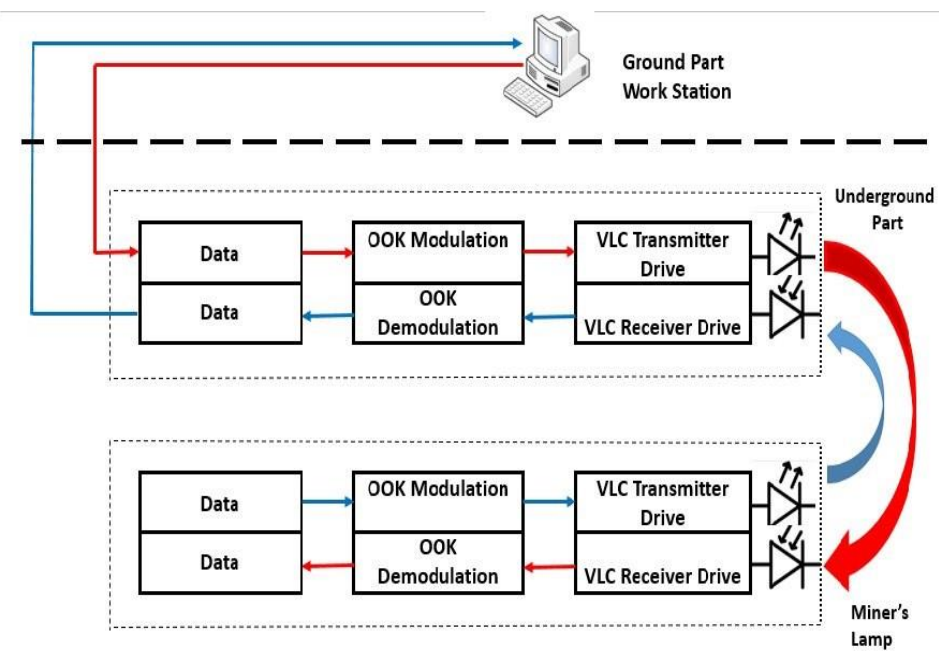

Figure 2. Block diagram of the proposed communication system.

We considered IM/DD approach which is widely used due to its implementation simplicity. At the transmitter, the information-bearing digital signal is converted to an electrical current to which a DC current is added to ensure a unipolar (positive-value) waveform. The resulting signal is then used to drive the LED.

At the receiver, PD collects the incident optical photons that falls in its FOV and generates an electrical current. This resulting current is then converted to a voltage using a transimpedance amplifier (TIA), which is then filtered to reduce the noise level and digitized prior to signal demodulation.

Also, in this model, we consider number of reflectors such as the underground mine walls, and the equipment in the mine. Background light noise interference exhibits a major limitation on the VLC system data rate. As PD is exposed to both the useful optical signal and the artificial illumination, an unwanted optical noise interference is present.

2) Channel Modeling: Optical wireless channel with noise is modelled as [19]

$Y(t)=\gamma X(t) \otimes h(t)+n(t) \quad(4)$

where $X(t)$ denotes the transmitted signal and $Y(t)$ represents the received signals, respectively, $\gamma$ denotes the conversion efficiency between optical and electrical signals, $h(t)$ denotes the channel impulse response, $\otimes$ means convolution and $n(t)$ denotes the Gaussian noise.

The horizontal illumination of the underground mine is given by:

$$
E=\frac{I_{0} \cos ^{m}(\varphi) \cos (\theta)}{d^{2}}
$$


where $I_{o}$ is the luminance value of the LED, $\varphi$ is the emission angle, $\theta$ is the incident angle, $m$ is Lambertian number, and $d$ is the distance between the transmitter and receiver. The distribution of horizontal illumination at the ground of lane is shown in Fig. 3.

We consider both LOS and NLOS paths for our signal communication and their impulse responses $h_{L O S}(t), h_{N L O S}(t)$ are given by Equations (2) and (3) respectively in [20]. The total impulse response can be given as

$$
h_{\text {Total }}(t)=h_{L O S}(t)+h_{N L O S}(t)
$$

In this work we have assumed one reflection system, as the received power for the high order reflection system is almost zero as can be seen from Fig. 4.

The received power for LOS and NLOS paths $\mathrm{Pr}^{L O S}, \mathrm{Pr}^{N L O S}$ are given by Equations (4) and (5) respectively in [20]. The total received power is given by

$$
P_{R}=P_{r}^{L O S}+P_{r}^{N L O S}
$$

At the receiver the electrical signal-to-noise ratio (SNR) is given by [19]:

$$
S N R=\frac{\left(\gamma P_{R}\right)^{2}}{\sigma_{\text {total }}^{2}}(8)
$$

where $\sigma_{\text {total }}^{2}$ is the total noise power produced by other artificial light sources in the mine.

\section{RESULTS AND DISCUSSION}

We used MATLAB software for our analysis, the parameters used in the simulations are listed in Table.1. We used the high-performance XM-L LED, which is usually recommended for underground lighting and is widely used. It can deliver 1000 lumens with 100 lumens per watt efficacy and is considered as best among white LED class. Its $V_{t o n}=2.7 \mathrm{~V}$ and maximum forward voltage is $V_{\max }=3.4 \mathrm{~V}$ [21]. We considered various underground challenges such as: shadowing, dust concentration, and propagation velocity. LED arrays illumination intensity as a function of distance is shown in Fig. 3, where it could help in the designing

\begin{tabular}{|c|c|c|}
\hline Parameter & Symbol & value \\
\hline Center luminous intensity/(cd) & $I$ & 1000 \\
\hline Transmitted optical power/(W) & $P_{t}$ & 8 \\
\hline Semi-angle at half power/(o) & $\varphi_{1 / 2}$ & 60 \\
\hline Detector's area $/ \mathrm{cm}^{2}$ & $A$ & 1 \\
\hline Photodiode responsivity & $\gamma$ & $\begin{array}{l}0.54 \\
\mathrm{~A} / \mathrm{W}\end{array}$ \\
\hline FOV at a receiver/(o) & $\Psi$ & 60 \\
\hline Distance between transmitter and receiver & $T_{s r}$ & $\begin{array}{c}30-100 \\
\mathrm{~m}\end{array}$ \\
\hline Dust concentration $/\left(\mathrm{mg} / \mathrm{m}^{3}\right)$ & $\mathrm{C}$ & 0.9 \\
\hline Miner's height/(m) & $\mathrm{L}$ & 2 \\
\hline Noise power & $\sigma_{\text {total }}^{2}$ & $10-12$ \\
\hline Scattering coefficient $/ \mathrm{m}$ & $\mathrm{Q}_{\mathrm{sca}}$ & 1.4115 \\
\hline Absorption coefficient/m & $\mathrm{Q}_{\mathrm{abs}}$ & 0.701 \\
\hline Lambertian number & $\mathrm{m}$ & 1 \\
\hline Mine wall reflection coefficient & $\rho$ & 0.4 \\
\hline Incidence angle & $\alpha$ & 30 \\
\hline Irradiance angle & $\beta$ & 45 \\
\hline Mining roadway dimensions in (m) & & $2.5 \times 6 \times 4$ \\
\hline Mining roadway radius in $(\mathrm{m})$ & & 1.5 \\
\hline Working face dimensions in $(\mathrm{m})$ & & $3 \times 6 \times 5$ \\
\hline Hight of miner's helmet in (m) & & 1.7 \\
\hline Number of LEDs & & 600 \\
\hline Number of reflectors & & 5 \\
\hline
\end{tabular}
stage of the underground mine lighting.

Table. 1 Proposed model simulation parameters

IJEEI, Vol. 8, No. 2, June 2020: $331-339$ 
1. Trajectory and Received Power: In Fig. 4, we show various signal paths and corresponding received power. Total received power is plotted versus communication distance. We plot both LOS and NLOS path powers and it is evident that, the dominant path in the VLC system is the LOS. This result shows high degree of agreement with our assumption in this work of considering one reflection system.

2. Shadowing versus BER: In Fig. 5, is plotted BER versus SNR, for various types of shadowing effect. The result shows the lower the degree of shadowing better is the system performance. Moreover, Fig. 5 shows that, the BER of the proposed system is between $\left(10^{-4}-10^{-6}\right)$ which is considered a good value to make the communication link stable. Also, we compared our results with the results given by [22] where the results showed the superiority of our system specially in mild and moderate shadowing cases, from Fig. 5 and for high SNR the BER $<10^{-5}$, which is considered a good value because it makes the communication link stable.

3. Channel Impulse Response: We showed in Fig. 6 the CIR for mining roadway and work face areas for LOS and NLOS paths. For roadway area, two overlapping peaks can easily be noticed. We used $h_{\text {LOS }}(t)$ impulse response to obtain CIR for LOS path (first peak) and identified as a delta function, scaled by $6.5 \times 10^{-6}$. While, the first-order response is shown by the second peak, corresponding to the ceiling and two walls of the mining roadway. The first-order CIR is $1.2 \times 10^{-6}$ which is obtained from $\mathrm{h}_{\mathrm{NLOS}}(\mathrm{t})$. In the context of first order as shown in Fig. 6 (a), a small early peak (close to the arrival time of LOS) can be seen in Fig. 6 (a). This peak shows power which arrives from the semi-cylindrical part. In terms of working face environment, CIR is shown in Fig. 6 (b). Here, the CIR of the LOS and first-order components are $1.3 \times 10^{-6}$ and $1.1 \times 10^{-6}$, respectively. This is because the width of the underground mining is only $3 \mathrm{~m}$ (not too wide). In terms of CIR, we compared our results with [9], where high degree of agreement is present.

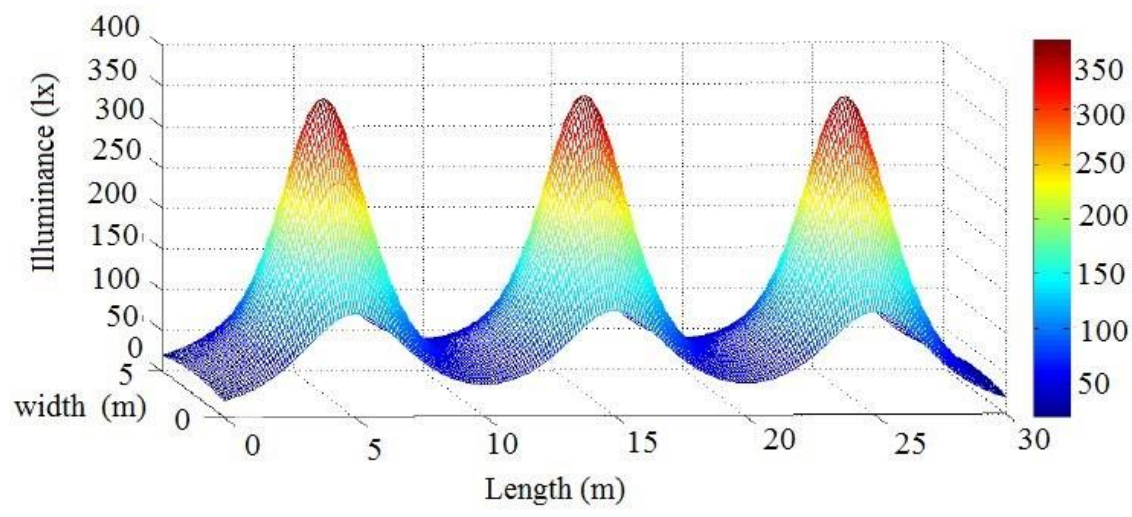

Figure 3. The distribution of horizontal illumination [16].

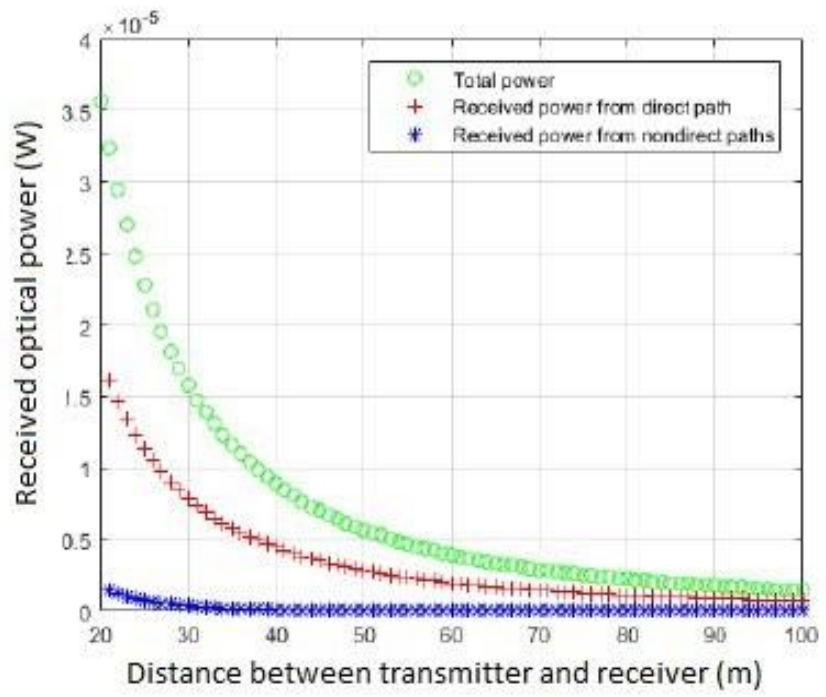

Figure 4. Received power vs the distance between the transmitter and receiver. 


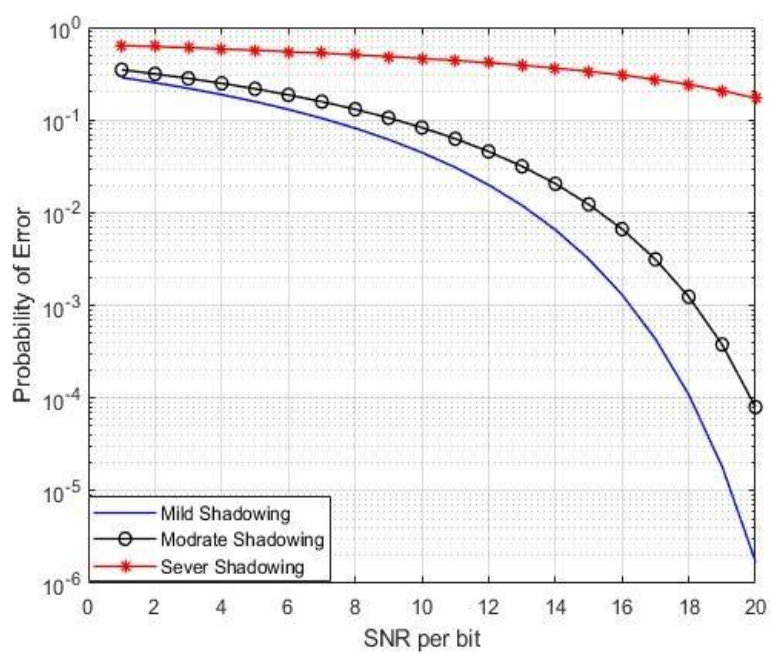

Figure 5. Error Probability with Different Shadowing Effects
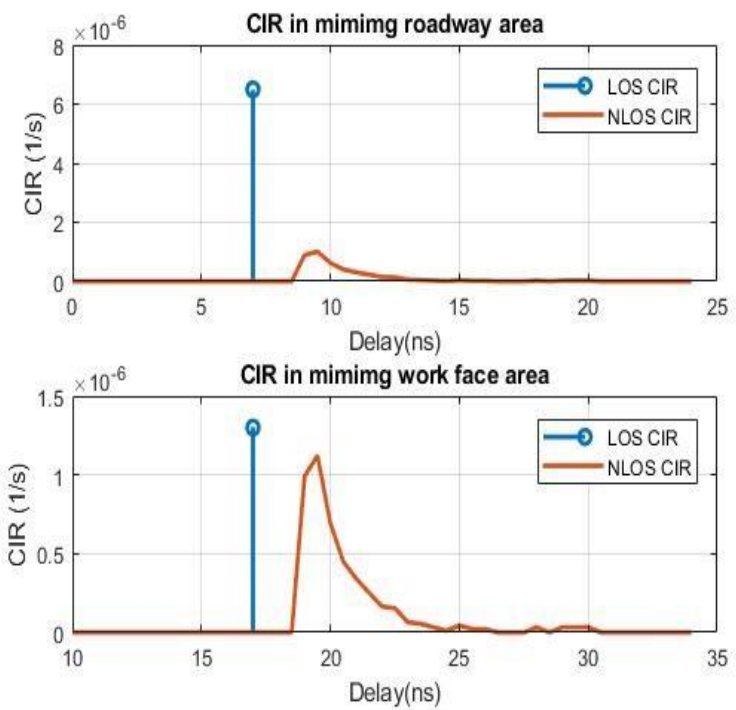

Figure 6. (a) Channel impulse response in mining roadway, (b)Channel impulse response in working face.

\section{CONCLUSION}

We have proposed and presented a visible light communication system for underground mining environment. This system is used to provide communication facilities as well as illumination for mine operations. It is comprised of LEDs, PDs and VLC enabled miner's light. We have modelled the optical communication channel and considered practical limitations of such system, i.e., reflectors, shadowing, dust effect, and interferers. Simulation results showed that system performance is greatly affected by shadowing, and mine dust particles, where the BER can be degraded to 10-2 in severe shadowing case which affects the stability of the communication link. We showed our system evaluation by plotting error probability and impulse response. We observed that most of the signal strength in mining environment is contained in LOS signal and the first reflector part of the signal. Finally, we compared our proposed system performance with others in literature, where our system showed good improvements.

\section{REFERENCES}

[1] P. A. Haigh, Z. Ghassemlooy, S. Rajbhandari, and I. Papakonstantinou, "Visible light communications using organic light emitting diodes," IEEE Communications Magazine, vol. 51, pp. 148-154, August 2013.

[2] R. O. H. Anis, P. M. Tardif and P. Fortier, "Communications network for underground mines based on the ieee 802.11 and docsis standards," in in Proc. IEEE 60th Vehicular Technology Conference (VTC2004Fall), Los Angeles, California, U.S.A., pp. 3605-3609, Sept 2004.

[3] P. K. M. L. K. Bondyopadhyay, S. K. Chaulya, "Wireless communication in underground mines," Springer Publication, 2010.

IJEEI, Vol. 8, No. 2, June 2020: $331-339$ 
[4] U. M. C. (in four parts), "Paging system/compiled by staff-mining research," Pittsburgh Mining and Safety Research Centre. [Washington]: United States Department of the Interior, Bureau of Mines, 1977.

[5] B. A. Austin, "Medium frequency body loop antenna for use underground," IEE Colloquium on Electrically Small Antennas, Oct 1990.

[6] D. Ghosh, H. Moon, and T. K. Sarkar, "Design of through-the-earth mine communication system using helical antennas," in 2008 IEEE Antennas and Propagation Society International Symposium, pp. 1-4, July 2008.

[7] P. M. L.K. Bandyopadhyay, S.K. Chaulya, "Wireless communication in underground mines : Rfid-based sensor networking," New York; London : Springer, 2009.

[8] Trong-Hop Do and Myungsik Yoo, "An in-depth survey of visible light communication based positioning systems," Sensors, 2016.

[9] J. Wang, A. Al-Kinani, W. Zhang, and C. X. Wang, "A new vlc channel model for underground mining environments," in 2017 13th International Wireless Communications and Mobile Computing Conference (IWCMC), pp. 2134-2139, June 2017.

[10] C. D. J. Statham, "Underground lighting in coal mine," in in Proc. IEEPart A: Power Engineering, vol. 103, pp. 396-409, August 1956.

[11] T. Komine and M. Nakagawa, "A study of shadowing on indoor visiblelight wireless communication utilizing plural white led lightings," in 1st International Symposium onWireless Communication Systems, 2004., pp. 36-40, 2004.

[12] H. Farahneh, C. Mekhiel, A. Khalifeh, W. Farjow, and X. Fernando, "Shadowing effects on visible light communication channels," in 2016 IEEE Canadian Conference on Electrical and Computer Engineering (CCECE), pp. 1-5, 2016.

[13] N. H. B. P. Catherine Forbes, Merran Evans, "Statistical Distributions,4th Edition," in New York: Jhon Willy, 2005., 2005, ISBN 978-0470390634.

[14] W. Y.-M. S. G.-Q. Wen-Zheng, Wang and W. De-Ming, "Numerical study on infrared optical property of diffuse coal particles in mine fully mechanized working combined with cfd method," Mathematical Problems in Engineering, 2015.

[15] J.-Z. Hong Tang, "Retrieval of spheroid particle size distribution from spectral extinction data in the independent mode using pca approach," Journal of Quantitative Spectroscopy and Radiative Transfer, vol. 115, pp. 78-92, 2013.

[16] J. Z. Gang Wu, "Demonstration of a visible light communication system for underground mining applications," in International Conference on Information Engineering and Communications Technology (IECT 2016), 2016.

[17] V. N. D. A. Viljoen and M, "Is there a risk to safety when working in the new south wales underground coalmining industry while having binaural noise-induced hearing loss?" Internal Medicine Journal, vol. 36, 2006.

[18] A. Ranjan, P. Misra, B. Dwivedi, and H. B. Sahu, "Studies on propagation characteristics of radio waves for wireless networks in underground coal mines," Wireless Personal Communications, vol. 97, pp. 2819-2832, Nov 2017.

[19] J. B. J.M. Kahn, "Wireless infrared communications," Proceeding of the IEEE, vol. 85, pp. 265-298, Feb 1997.

[20] H. Farahneh, F. Hussain, and X. Fernando, "A new alarming system for an underground mining environment using visible light communications," in 2017 IEEE Canada International Humanitarian Technology Conference (IHTC), pp. 213-217, July 2017.

[21] "www.digikey.com/catalog/en/partgroup/xlamp-xm-l-leds/12997@ONLINE."

[22] P. Athar Ravish Khan, Sanjay M. Gulhane, "The bit error rate analysis of direct sequence ultra-wide band system in mine channel," International Journal of Advancements in Technology, vol. 3, pp. 146-156, July 2012.

\section{BIOGRAPHIES OF AUTHORS}

Ibrahim Mansour: imansour@ammanu.edu.jo is an associated professor at Al-Ahliyya Amman university. His research interest includes optical communication and free space optical communications. 